\title{
Search for the magnetic field of the O7.5 III star $\xi$ Persei
}

\author{
Huib F. Henrichs ${ }^{1}$, R.S. Schnerr ${ }^{2}$, J.A. de Jong ${ }^{3}$, L. Kaper ${ }^{1}$, \\ J.-F. Donati ${ }^{4}$ and C. Catala ${ }^{5}$ \\ ${ }^{1}$ Astronomical Institute, University of Amsterdam, Amsterdam, Netherlands \\ ${ }^{2}$ Inst. for Solar Physics, Royal Swedish Academy of Sciences, Stockholm, Sweden \\ ${ }^{3}$ Max Planck Institute for Extraterrestrial Physics, Garching, Germany \\ ${ }^{4}$ Observatoire Midi-Pyrénées, Toulouse, France \\ ${ }^{5}$ LESIA, Observatoire de Paris, CNRS, Université Paris Diderot, Meudon, France
}

\begin{abstract}
Cyclical wind variability is an ubiquitous but as yet unexplained feature among OB stars. The O7.5 III(n)((f)) star $\xi$ Persei is the brightest representative of this class on the Northern hemisphere. As its prominent cyclical wind properties vary on a rotational time scale ( 2 or 4 days) the star has been already for a long time a serious magnetic candidate. As the cause of this enigmatic behavior non-radial pulsations and/or a surface magnetic field are suggested. We present a preliminary report on our attempts to detect a magnetic field in this star with highresolution measurements obtained with the spectropolarimeter Narval at TBL, France during 2 observing runs of 5 nights in 2006 and 5 nights in 2007. Only upper limits could be obtained, even with the longest possible exposure times. If the star hosts a magnetic field, its surface strength should be less than about $300 \mathrm{G}$. This would still be enough to disturb the stellar wind significantly. From our new data it seems that the amplitude of the known non-radial pulsations has changed within less than a year, which needs further investigation.
\end{abstract}

Keywords. Stars: magnetic fields - techniques: polarimetric - stars: atmospheres - stars: $\xi$ Per - stars: early-type - stars: winds - outflows - stars: rotation - stars: pulsations

\section{Introduction}

Like many $\mathrm{O}$ and B stars, the $\mathrm{O} 7.5 \mathrm{III}(\mathrm{n})((\mathrm{f}))$ star $\xi$ Per shows very prominent cyclical wind variability in the UV resonance lines (Fig. 1a), manifested by discrete absorption components (DACs) which migrate from red to blue, and narrow when they approach (but not reach) the terminal velocity as measured from saturated wind profiles (e.g. Kaper et al. 1999). In $\xi$ Per the DAC period is $2.09 \mathrm{~d}$. Multiwavelength observations of a number of OB stars, including $\xi$ Per, have shown that the cyclic behavior is present down to the surface of the star (de Jong et al. 1997), and that the typical timescale varies with the (estimated) rotational timescale. This strongly argues in favor of a surface phenomenon which perturbs the base of the flow. Two scenarios are proposed: in the so-called Corotating Interaction Region (CIR) model a perturbation at the surface of the star causes a local increase (or decrease) of the radiative force driving the stellar wind, or surface magnetic fields may disturb the outflow, both resulting in a rotationally modulated stellar wind (see Cranmer and Owocki 1996). A number of coordinated UV and optical observations have confirmed the CIR model, including for the case of $\xi$ Per, but the origin of the perturbations is not known, which is one of the most challenging problems in stellar wind research of the last decades. We present here our most recent efforts to measure the magnetic field and the pulsation properties. 

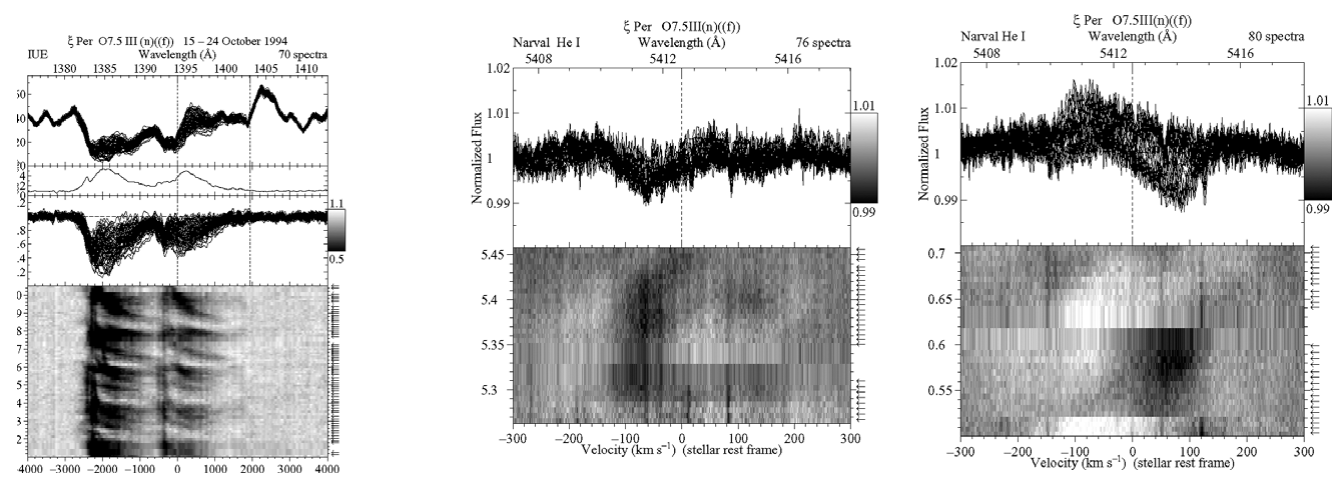

Figure 1. (a) Progressing DACs in the Si IV wind lines in 1994, (b) Non radial pulsation (period $3.5 \mathrm{~h}$ ) in the He I 5411 line, December 2006. (c) September 2007; the amplitude of the moving features seems to have changed after 9 months.
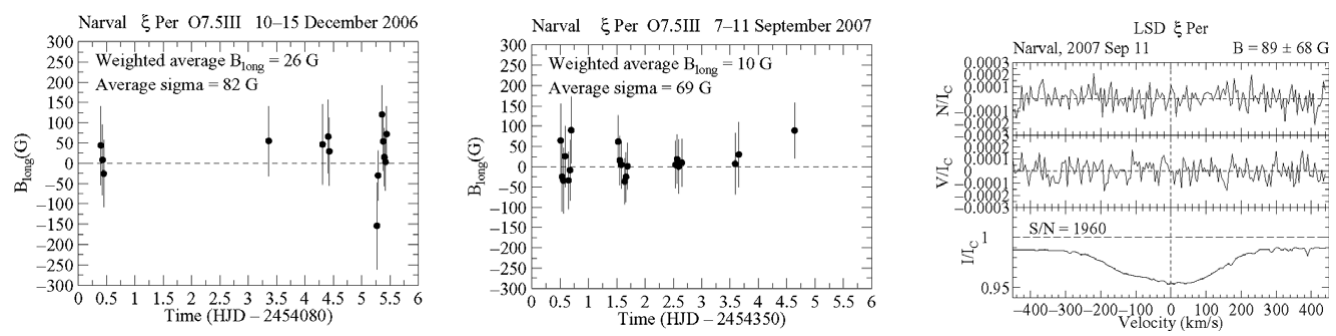

Figure 2. Preliminary magnetic results of Narval spectropolarimetry as a function of time. (a) December 2006. (b) September 2007. (c) LSD Stokes V profile of the last point in September 2007. No significant Zeeman signature was found.

\section{Data analysis and discussion}

Previous Musicos magnetic measurements of $\xi$ Per were presented by de Jong et al. (2001), with no detection. For Narval data the magnetic analysis is essentially the same, applying the least-squares deconvolution method (Donati et al. 1997) to the spectral lines sensitive to magnetic effects. This yields the longitudinal component of the field, averaged over the facing hemisphere of the star. In Fig. $2 \mathrm{a}$ and $2 \mathrm{~b}$ the 45 results are plotted as a function of time. No Zeeman signature was found. As an example, Fig. 2c shows the LSD profile of the best exposed spectrum of September 2007 with a $\mathrm{S} / \mathrm{N}=1960$. The magnetic values are preliminary, as the used spectral linelist can still be optimized.

We also analysed a number of spectral lines for non-radial pulsations with known period of $3.5 \mathrm{~h}$ found in a previous Musicos campaign (de Jong et al. 1999). The amplitude may have changed during the 9 months between our Narval runs, see Fig. 1b and 1c. This obviously needs further investigation, as the impact on the stellar wind may have changed.

Observations with a higher efficiency should be able to detect a possible field, unless the geometry is such that the magnetic properties of the two hemispheres cancel out.

\section{References}

de Jong, J. A., Henrichs, H. F., Schrijvers, C., Gies, D. R. et al. 1999, A\& A 345, 172

de Jong, J. A., Henrichs, H. F., Kaper, L., Nichols, J. S. et al. 2001, A $\varepsilon_{A} A$ 368, 601

Cranmer, S. R. \& Owocki, S. P. 1996, ApJ 462, 469

Donati, J.-F., Semel, M., Carter, B. D. et al. 1997, MNRAS 291, 658

Kaper, L., Henrichs, H. F., Nichols, J. S., \& Telting, J. H. 1999, A\&A 344, 231 